\title{
Integrated Fourier Transform Infrared Imaging and Proteomics for Identification of a Candidate Histochemical Biomarker in Bladder Cancer
}

Kathrin E. Witzke, ${ }^{*}$ Frederik Großerueschkamp, ${ }^{\dagger}$ Hendrik Jütte, ${ }^{\ddagger}$ Melanie Horn, ${ }^{\dagger}$ Florian Roghmann,${ }^{\S}$ Nicolas von Landenberg,

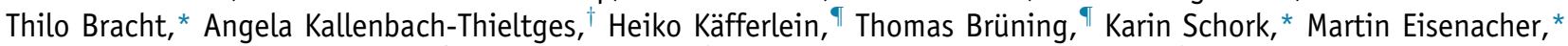
Katrin Marcus, ${ }^{*}$ Joachim Noldus, $^{\S}$ Andrea Tannapfel, ${ }^{\ddagger}$ Barbara Sitek, ${ }^{*}$ and Klaus Gerwert ${ }^{\dagger}$

From the Medizinisches Proteom-Center, ${ }^{*}$ the Department of Biophysics, ${ }^{\dagger}$ the Institute of Pathology, ${ }^{\ddagger}$ the Department of Urology, ${ }^{\S}$ Marien Hospital Herne, and the Institute for Prevention and Occupational Medicine of the German Social Accident Insurance, ${ }^{\top}$ Ruhr University Bochum, Bochum, Germany

\author{
Accepted for publication \\ November 26, 2018. \\ Address correspondence to \\ Klaus Gerwert, Dr.rer.nat., \\ Department of Biophysics; or \\ Barbara Sitek, Dr.rer.nat., \\ Medizinisches Proteom-Center, \\ Ruhr University Bochum, \\ Universitätsstrasse 150, 44801 \\ Bochum, Germany. E-mail: \\ gerwert@bph.ruhr-uni-bochum. \\ de or barbara.sitek@rub.de.
}

\begin{abstract}
Histopathological differentiation between severe urocystitis with reactive urothelial atypia and carcinoma in situ (CIS) can be difficult, particularly after a treatment that deliberately induces an inflammatory reaction, such as intravesical instillation of Bacillus Calmette-Guèrin. However, precise grading in bladder cancer is critical for therapeutic decision making and thus requires reliable immunohistochemical biomarkers. Herein, an exemplary potential biomarker in bladder cancer was identified by the novel approach of Fourier transform infrared imaging for label-free tissue annotation of tissue thin sections. Identified regions of interest are collected by laser microdissection to provide homogeneous samples for liquid chromatography-tandem mass spectrometry-based proteomic analysis. This approach afforded label-free spatial classification with a high accuracy and without interobserver variability, along with the molecular resolution of the proteomic analysis. Cystitis and invasive highgrade urothelial carcinoma samples were analyzed. Three candidate biomarkers were identified and verified by immunohistochemistry in a small cohort, including low-grade urothelial carcinoma samples. The best-performing candidate AHNAK2 was further evaluated in a much larger independent verification cohort that also included CIS samples. Reactive urothelial atypia and CIS were distinguishable on the basis of the expression of this newly identified and verified immunohistochemical biomarker, with a sensitivity of $97 \%$ and a specificity of $69 \%$. AHNAK2 can differentiate between reactive urothelial atypia in the setting of an acute or chronic cystitis and nonmuscle invasive-type CIS. (Am J Pathol 2019, 189: 619-631; https://doi.org/10.1016/j.ajpath.2018.11.018)
\end{abstract}

Bladder cancer is the second most common urogenital malignancy, with approximately 430,000 new cases diagnosed worldwide in 2012. . $^{1,2}$ Approximately $70 \%$ to $75 \%$ of patients are newly diagnosed with nonmuscle invasive, mostly low-grade bladder cancer, whereas $25 \%$ to $30 \%$ of de novo diagnoses are high-grade bladder cancer at the stage of infiltration of the muscularis, with metastases observed in $10 \%$ of cases. $^{3-5}$ A significant percentage $(50 \%$ to $70 \%$ ) of patients diagnosed with nonmuscle invasive cancer will have multiple recurrences, and $10 \%$ to $15 \%$ will even progress to invasion. ${ }^{3,6,7}$ A special case of bladder cancer is carcinoma in situ (CIS), which is a flat, noninvasive tumor with a rate of progression to an invasive state of approximately $54 \% .^{8}$
The prognosis of bladder cancer (BC) is stage and grade dependent; survival rate is higher for lower stages (T2 or less). ${ }^{6,9}$ Because there are currently no established biomarkers for bladder cancer, ${ }^{10}$ a diagnosis is established

\footnotetext{
Supported by the Ministry of Innovation, Science and Research of NorthRhine Westphalia, Germany, project 233-1.08.03.03-031.68079 (K.E.W., F.G., H.J., M.H., F.R., N.v.L., T.Bra., A.K.-T., H.K., T.Brü., K.S., M.E., K.M., J.N., A.T., B.S., and K.G.); and the German Federal Ministry of Education and Research, German Network for Bioinformatics Infrastructure (de.NBI), grant FKZ 031 A 534A (K.S. and M.E.)

K.E.W., F.G., and H.J. contributed equally to this work.

B.S. and K.G. contributed equally to this work as senior authors.

Disclosures: None declared.
} 
solely on the basis of microscopic examination of biopsied lesions. However, visual scoring of tumor grade and stage shows interobserver variability. ${ }^{11}$ The differentiation of reactive urothelial atypia as part of an acute or chronic urocystitis and CIS can be particularly difficult, especially after previous treatment with attenuated mycobacteria (Bacillus Calmette-Guèrin) for deliberate induction of an inflammatory reaction, which is a primary treatment option for CIS aside from early cystectomy. ${ }^{4}$ Correct discrimination of nonmuscle invasive low-grade bladder cancer and CIS is not trivial; in some cases, even differentiating between low- and invasive high-grade tumors can be challenging. ${ }^{12}$ There is therefore a need for a sensitive and specific biomarker that can support pathologic findings in the diagnosis of different subtypes of bladder cancer.

Extensive proteomics studies of urine, serum, and tissue samples have been performed to identify bladder cancer biomarkers, ${ }^{13-17}$ and candidate proteins have been proposed as immunohistochemical markers for diagnosis and prognosis, including CD44, p53, and cytokeratin CK20. p53 Accumulation is correlated with tumor stage and grade and is observed in invasive high-grade tumors, ${ }^{18}$ whereas cytokeratin CK20 is up-regulated early in abnormal urothelial differentiation and is therefore also a biomarker for lowgrade tumors. ${ }^{19}$ The use of an immunohistochemistry panel consisting of CD44, cytokeratin CK20, and p53 has been proposed to differentiate between benign and neoplastic entities. ${ }^{20}$ However, in practice, categorizing an immunohistochemical stain as strictly positive or negative can be difficult, a fact that is further aggravated by trying to rely on a three-marker panel. A reliable single marker to differentiate benign from neoplastic flat urothelial lesions is not found yet. Visual inspection of a hematoxylin and eosin (H\&E)-stained tissue section remains the gold standard for tumor grading and staging.

Given the limitations of earlier attempts to identify appropriate biomarkers for bladder cancer, a novel approach was used in the present study. To limit interfering factors and use only homogeneous cases of severe urocystitis or invasive highgrade carcinoma tissue samples, laser capture microdissection (LCM) was used to obtain a sample from a region of interest (ROI). Mass spectrometry (MS) combined with LCM has been successfully applied to the identification of candidate biomarkers in various cancers, ${ }^{21-24}$ including bladder cancer. ${ }^{25}$ However, to date, it has not been possible to use label-free annotated tissue samples with minimal interobserver and intraobserver variation for liquid chromatography-tandem MS (LC-MS/MS) analyses. Typically, histologic or pathologic examinations are necessary to delineate ROIs in tissue samples, which are then transferred to unstained adjacent sections for LCM. However, this invariably leads to deviations in annotated ROIs because these will only partly overlap with diseased tissue annotated by the pathologist, resulting in more or less heterogeneous samples.

To circumvent this problem, label-free Fourier transform infrared (FTIR) - guided LCM was used to obtain specimens for subsequent proteomic analysis. This approach was recently implemented in a validation study of biomarkers in immunohistochemical panels to differentiate between pleural mesothelioma subtypes. ${ }^{26}$ Tissue classification by FTIR imaging allows label-free annotation of thin sections with high accuracy ( $>90 \%$ sensitivity and specificity) and without interobserver variability as opposed to pathologic annotation. ${ }^{27-31}$ Differential analyses can also be performed by label-free FTIR imaging, as demonstrated for lung, colon, and breast cancer. ${ }^{3}$

As a first step, an IR classifier for bladder tissue was developed to identify ROIs. Ten invasive high-grade bladder cancer samples with no accompanying CIS and seven cystitis tissue samples were classified label free by FTIR imaging. This classification was subsequently transferred to an LCM microscope to collect label-free samples of the same thin tissue section for subsequent LC-MS/MS analysis. This yielded more homogeneous samples for proteomic analysis than would be obtained from adjacent sections. The proteomics analysis revealed three candidate biomarkers that were then verified in the discovery phase by immunohistochemistry (IHC) in three sets of samples, including 20 invasive high-grade and 20 cystitis formalinfixed, paraffin-embedded (FFPE) tissue specimens as well as 20 low-grade samples. In a third step, the best-performing candidate biomarker from the discovery IHC study, AHNAK2, was further verified in a second independent and much larger cohort of four groups, including FFPE tissue samples of 51 high-grade muscle invasive BC, 67 CIS, 84 low-grade $\mathrm{BC}$, and 108 patients with severe cystitis containing reactive urothelial atypia, low grade. AHNAK2 was found to achieve $97 \%$ sensitivity and $69 \%$ specificity in differentiating between severe cystitis with reactive urothelial atypia (RUA) and CIS; 80\% sensitivity and 86\% specificity in differentiating between low and invasive high grades; and $97 \%$ sensitivity and $55 \%$ specificity in differentiating between low grade and CIS.

\section{Materials and Methods}

\section{Sample Sets}

Five sample sets were used in this study. Sample set 1 $(N=11)$ was the training set for the FTIR imaging random forest (RF) classifier and included invasive high-grade $(n=6)$, low-grade $(n=3)$, and severe cystitis with RUA $(n=2)$ specimens. Sample set $2(N=103)$ was used for verification of the FTIR imaging tissue classification and comprised freshly frozen samples of severe cystitis with RUA $(n=41)$, low-grade carcinoma $(n=19)$, and invasive high-grade carcinoma $(n=43)$. For the discovery phase, sample set 3 was used. Sample set $3(N=17)$ was used for combined FTIR imaging and label-free LC-MS/MS and included freshly frozen tissue from invasive high-grade $(n=10)$ and severe cystitis with RUA $(n=7)$ patients who were between 48 and 85 years of age, with a mean age 
of 67 years. Of the 17 patients, 4 were female and 13 were male (Supplemental Table S1). Sample set $4(N=60)$ for the first candidate biomarker verification step included FFPE tissues from severe cystitis with RUA and invasive highgrade and also low-grade patients $(n=20$ each), all male and between 60 and 80 years of age (mean, 70 years) (Supplemental Table S2). Sample set $5(N=310)$ for the second candidate biomarker verification step consisted of FFPE tissues from severe cystitis with RUA $(n=108)$, invasive high-grade without CIS $(n=51)$, low-grade $(n=84)$, and CIS $(n=67)$ patients between 23 and 90 years of age, with a mean age of 69 years (Supplemental Table S3).

Because urothelial dysplasia is not fully understood and its significance is unclear, it was not included in this analysis.

\section{Clinical Data}

Sample sets 1 to 4 as well as some high-grade bladder cancer patients $(n=20)$ from sample set 5 were collected at the Department of Urology, Ruhr University Bochum, Marien Hospital Herne (Bochum, Germany), and were managed and distributed by the Institute for Prevention and Occupational Medicine of the German Social Accident Insurance (Ruhr University Bochum). The local ethics committee approved the study (approval number 3674-10). The remaining specimens in sample set 5 were directly obtained at the Department of Urology, Ruhr University Bochum, Marien Hospital Herne, with approval from the local ethics committee (approval number 4047-11). Written, informed consent was obtained from each patient, and the study protocol conformed to the ethical guidelines of the 1975 Declaration of Helsinki.

\section{Sample Preparation for Discovery}

Sample sets 1 to 3 for the discovery phase were collected during surgery, according to a standard operation procedure. The samples were washed with isotonic saline solution, slowly frozen on the surface of liquid nitrogen within 8 minutes, and stored at $-80^{\circ} \mathrm{C}$ until further processing. Frozen sections were obtained using an HM550 cryostat (Thermo Fisher Scientific, Waltham, MA) at $-20^{\circ} \mathrm{C}$. Sections cut at a thickness of $10 \mu \mathrm{m}$ were collected on polyethylene terephthalate frame slides. The cryostat was cleaned between samples with $70 \%$ ethanol to avoid crosscontamination. All preparatory steps were performed as quickly as possible, according to standard operation procedures, to guarantee high-quality samples with minimal tissue and protein degradation.

\section{FTIR Imaging}

FTIR imaging spectroscopy was performed in reflection mode on low-e microscope slides (Kevley Technologies,
Chesterland, $\mathrm{OH}$ ) or in transmission mode on polyethylene terephthalate frame slides using a Cary 620 IR microscope (Agilent Technologies, Santa Clara, CA) equipped with a $128 \times 128$-pixel liquid nitrogen-cooled mercury cadmium telluride focal plane array detector. The collected wave number region was 3700 to $950 \mathrm{~cm}^{-1}$ at a spectral resolution of $4 \mathrm{~cm}^{-1}$. For sample and background spectra, 128 scans were coadded. The mapped pixel resolution was approximately $5.5 \mu \mathrm{m}$ for a tissue sampling area of approximately $715 \mu \mathrm{m}^{2}$ for each focal plane array field. This resulted in oversampling due to a lower optical resolution in the IR region. The results of this and previous studies have shown that oversampling does not affect cancer detection. ${ }^{35}$ The Cary 670 spectrometer (Agilent Technologies), microscope, and sample chamber were continuously flushed with dry air to avoid spectral contributions from atmospheric water. In addition, a 24/7 liquid nitrogen cooling supply (Norhof, Maarssen, the Netherlands) was included in the system, permitting continuous measurement. FT was performed with Mertz phase correction and Blackman-Harris four-term apodization. Measurements were made in the mosaic mode of Agilent Resolutions Pro software version 5.3.0.1694. Individual mosaic tiles, each measuring $128 \times 128$ pixels, were stitched together automatically after the measurement. Each raw spectral vector consisted of 1428 data points (resolution, $4 \mathrm{~cm}^{-1}$; zero filling, 4; upper folding limit, $\left.5266 \mathrm{~cm}^{-1}\right)$. The stitching was performed in Matlab 2017b (MathWorks, Natick, MA). The RF was established on low-e slides and extended to polyethylene terephthalate frame slides for FTIR-guided LCM.

\section{Spectral Preprocessing and Analysis}

Preprocessing of the raw data sets was performed in Matlab as follows; it is possible to work with an oversampled data set collected at $5.5-\mu \mathrm{m}$ spatial resolution. ${ }^{35}$ All images presented herein are from the original data set of unbinned data. The first step after stitching was a quality test based on the integral of the amide I band and the signal/noise ratio (noise, 2100 to $2000 \mathrm{~cm}^{-1}$; signal, 1600 to $1500 \mathrm{~cm}^{-1}$ ). Pixel spectra from the vicinity of voids or cracks in the tissue and regions without tissue were removed in this step. ${ }^{36}$ All spectra were then subjected to extended multiplicative scattering correction-based Mie and resonanceMie scattering correction from 2300 to $950 \mathrm{~cm}^{-1}$ in one iteration. ${ }^{37}$ Higher iteration numbers were tested, but because of low scattering effects, the classification results were not further improved. Thus, processing time was more important for this study than perfect spectral correction. The spectral range from 1800 to $950 \mathrm{~cm}^{-1}$ was used for unsupervised hierarchical and k-means clustering and the supervised RF algorithm. For unsupervised methods, the second derivative of the smoothed spectra was examined. The smoothing was performed using a nine-point SavitzkyGolay filter. ${ }^{38}$ The supervised RF was trained on 
unsmoothed absorption spectra. Tests with the second derivative did not result in higher accuracy for this classification.

\section{Coordinate Transfer}

The coordinates of ROIs selected from the FTIR imaging results were transferred to LCM by two-dimensional Helmert transformation on the basis of three reference points. Disparities in pixel size and rotation between the FTIR imaging microscope and LCM were adjusted. For this purpose, the FTIR imaging microscope was calibrated using a 1951 United States Airforce Target glass slide resolution target (USAF 1951 1×; Edmund Optics, Barrington, NJ). The resultant pixel size of the focal plane array detector was determined to be $5.65 \times 5.75 \mu \mathrm{m}$, and the rotation between the visible and IR image microscope was approximately 0.06 degrees. $^{26}$

\section{Selection of ROIs and LCM}

The results of the RF are presented in an index color image representing different tissue types, and the color representing the tissue of interest was defined as the ROI. The thin tissue section was classified according to its spectral fingerprint without further processing. Thus, FTIR-guided LCM enabled collection of label-free sample pools without interobserver and intraobserver variability. Samples of 1 $\mathrm{mm}^{2}$ were collected in $20 \mu \mathrm{L}$ of $50 \mathrm{mmol} / \mathrm{L}$ ammonium bicarbonate with $0.1 \%$ RapiGest SF surfactant (Waters $\mathrm{GmbH}$, Eschborn, Germany) for cell lysis. LCM was performed using a Zeiss (Oberkochen, Germany) Palm system. Samples were stored at $-80^{\circ} \mathrm{C}$ until further processing.

\section{Histologic Analysis}

$\mathrm{H} \& \mathrm{E}$ staining was performed on the same slides that were previously used for FTIR spectroscopy analysis. Tissue samples were washed with Milli-Q ultrapure water (Merck Millipore, Darmstadt, Germany), stained with Harris hematoxylin (VWR, Bruchsal, Germany) for 50 seconds, washed with water, counterstained with eosin (Merck Millipore), dehydrated in increasing gradients of alcohol, and mounted with Euparal (Carl Roth GmbH, Karlsruhe, Germany). Stained sections were imaged automatically with a BX41 microscope (Olympus, Tokyo, Japan).

\section{Sample Preparation for LC-MS/MS Analysis}

LCM vials were sonicated upside down on ice for 1 minute and then centrifuged for 1 minute in the upright position to transfer the sample from the lid into the vial itself. A $3.7-\mu \mathrm{L}$ volume of dithiothreitol $(20 \mathrm{mmol} / \mathrm{L})$ was added to the sample for reduction $\left(30\right.$ minutes, $60^{\circ} \mathrm{C}$ ), followed by 2.2 $\mu \mathrm{L}$ iodoacetic acid ( $100 \mathrm{mmol} / \mathrm{L}$ ) for alkylation (30 minutes, room temperature in the dark). Lysed proteins were tryptically digested overnight at $37^{\circ} \mathrm{C}(0.6 \mu \mathrm{L}$ trypsin; 0.033 $\mu \mathrm{g} / \mu \mathrm{L})$. For acidification, $1.3 \mu \mathrm{L}$ of trifluoroacetic acid $(10 \%)$ was added $\left(30\right.$ minutes, $\left.37^{\circ} \mathrm{C}\right)$, and samples were transferred to glass vials, dried in a vacuum centrifuge, and dissolved in $0.1 \%$ trifluoroacetic acid. A sample amount corresponding to an approximately $0.5-\mathrm{mm}^{2}$ area of laser microdissected tissue was used for one MS measurement.

\section{LC-MS/MS Analysis}

LC-MS/MS analysis was performed on an LTQ Orbitrap Elite instrument (Thermo Fisher Scientific) coupled online to an upstream-connected Ultimate 3000 RSLCnano highperformance liquid chromatography system (Dionex, Idstein, Germany), as previously described. ${ }^{39}$ Briefly, preconcentration of peptides was performed on a C18 trap column (Acclaim PepMap 100; $100 \mu \mathrm{m} \times 2 \mathrm{~cm}, 5 \mu \mathrm{m}, 100$ $\AA$ A Thermo Fisher Scientific) within 7 minutes at a flow rate of $30 \mu \mathrm{L} /$ minute with $0.1 \%$ trifluoroacetic acid. The peptides were then transferred to a Nano Viper C18 analytical column (Acclaim PepMap RSLC; $75 \mu \mathrm{m} \times 50 \mathrm{~cm}, 2 \mu \mathrm{m}$, $100 \AA$ A Thermo Fisher Scientific). Peptide separation was achieved with a gradient from 5\% to $40 \%$ solvent B over 98 minutes at $400 \mathrm{~nL} /$ minute and $60^{\circ} \mathrm{C}$ (solvent $\mathrm{A}, 0.1 \%$ formic acid; solvent B, $0.1 \%$ formic acid and $84 \%$ acetonitrile). MS/MS spectra were acquired in data-dependent mode. Full-scan mass spectra in the Orbitrap analyzer were acquired in profile mode at a resolution of 60,000 at $400 \mathrm{~m} / \mathrm{z}$ and within a mass range of 350 to $2000 \mathrm{~m} / \mathrm{z}$. For MS/MS measurements, the 20 most abundant peptide ions were fragmented by collision-induced dissociation and measured for tandem mass spectra in the linear ion trap.

\section{Protein Identification and Quantification}

Proteins were identified using Proteome Discoverer version 1.4 (Thermo Fisher Scientific). The spectra were searched against the UniProtKB/Swiss-Prot database (release 2015_10; 70,075 entries) using Mascot version 2.5 (Matrix Science, London, UK). The taxonomy setting was Homo sapiens, and mass tolerance was $5 \mathrm{ppm}$ and $0.4 \mathrm{Da}$ for precursor and fragment ions, respectively. Dynamic and static modifications were considered for methionine (oxidation) and cysteine (carbamidomethyl), respectively. The false discovery rate (FDR) was calculated with the Proteome Discoverer Percolator function, and identifications with an FDR $>1 \%$ were rejected. The software Progenesis QI version 2.0.5387.52102 (Nonlinear Dynamics, Durham, NC) was used for label-free quantification. The obtained .raw files were aligned to a reference run, and a master map of common features was applied to all experimental runs to adjust for differences in retention time. Peak picking in Progenesis was performed in default mode, where a feature is only generated when the corresponding peak is present in most experimental runs. If no peak is detected in a minority of experimental runs, the value for the feature in the 
corresponding runs is set to 0 . Ion charge states of $2+, 3+$, and $4+$ with a minimum of three isotope peaks were considered, and raw ion abundances were normalized for automatic correction of technical or experimental variations between runs. ${ }^{40}$ Quantified features were identified using the obtained Proteome Discoverer identifications. All nonconflicting peptides were considered for protein quantification. An in-house $\mathrm{R}$ script ( $\mathrm{R}$ version 3.4 .0$)^{41}$ was applied to the normalized protein abundances obtained from the software for $t$-test calculations. Ratios of means between groups were determined on the basis of normalized abundances, whereas $t$-test calculations used arcsinh-transformed data to maintain consistency with Progenesis QI software. $t$ Test $P$ values were adjusted for FDR control $\left(p_{\mathrm{FDR}}\right)$ with the method of Benjamini and Hochberg. ${ }^{42}$ Proteins were considered as differentially abundant between groups when the absolute ratio of means was $\geq 1.5$ and the $p_{\mathrm{FDR}}$ was $\leq 0.05$. Proteomics data have been deposited as a complete submission in the ProteomeXchange Consortium via the PRIDE partner repository (http://www.proteomexchange. org, last accessed August 24, 2018; data set identifier: PXD009203). The .msf files obtained in Proteome Discoverer were converted into the mzIdentML standard format using ProCon PROteomics Conversion tool version $0.9 .718 .^{43}$

\section{Verification by IHC}

IHC was performed as previously described. ${ }^{44}$ Samples were fixed in $4 \%$ formaldehyde solution and embedded in paraffin. Sections were prepared at a thickness of $4 \mu \mathrm{m}$, deparaffinized in xylene, and then rehydrated. Endogenous peroxidase activity was blocked for 30 minutes with 3\% hydrogen peroxide in methanol. After a short rinse in phosphate-buffered saline, sections were preincubated with avidin-biotin (SP-2001; Vector Laboratories, Burlingame, CA) for 15 minutes to reduce non-specific background staining. The sections were incubated with normal goat serum for 20 minutes, followed by primary antibodies against cytokeratin CK5/6 (Dako, Glostrup, Denmark; M7237) at 1:500; asporin (ASPN; Abcam, Cambridge, MA; ab201208) at 1:100; and AHNAK2 (Atlas Antibodies,
Bromma, Sweden; HPA004145) at 1:1000. After washing with phosphate-buffered saline, the sections were incubated with biotinylated goat anti-mouse $\operatorname{IgG}$ (BioGenex, Hamburg, Germany) for 30 minutes and then with peroxidase-conjugated streptavidin (Dako). The peroxidase reaction was allowed to proceed for 8 minutes, with $0.05 \%$ 3,3-diaminobenzidine tetrahydrochloride solution added as substrate. Sections were counterstained with hematoxylin. Negative controls were prepared by replacing the primary antibody with mouse or goat ascites fluid (Sigma-Aldrich, St. Louis, MO). The slides were examined and scored. At least eight randomly selected $\times 20$ high-power fields with a minimum of 4000 cells were scored for degree of expression. The staining in tumor cells was scored on the basis of the immunoreactivity score (IRS). ${ }^{45}$ Intensity was measured from 0 (no reaction) to 3 (strong reaction). The relative proportion of positive tumor cells was scored as follows: 0 (no reaction), 1 ( $<10 \%$ expression), 2 (10\% to $50 \%$ expression), 3 (51\% to $80 \%$ expression), and 4 (>80\% expression). The two individual results were then multiplied to obtain the IRS.

\section{Statistical Analysis}

Statistical analysis for verification was performed with $\mathrm{R}$ scripts written in house. For IRSs, nonparametric statistics were applied because of the discrete nature of their measurement. The Kruskal-Wallis test was used to identify overall differences in marker expression between groups. To account for multiple testing of different markers, $P$-value adjustment was applied using the Bonferroni method. When the result was significant, the Wilcoxon rank-sum test was used for pairwise comparisons of groups and the corresponding $P$ values were again adjusted by applying the Bonferroni correction. The power of the markers to distinguish between cystitis and different tumor grades was evaluated by receiver operating characteristic (ROC) curve analyses using the R package pROC ${ }^{46}$ which revealed the balance between sensitivity (true positive rate) and specificity (true negative rate) of a marker in a defined comparison. Values for area under the curve as well as corresponding 95\% CIs were determined. Youden's

Table 1 Sensitivity, Specificity, Accuracy, and Predictive Values for the Automated Detection of Bladder Carcinoma in Native Tissue by FTIR Imaging (Sample Set 2, $n=103$ )

\begin{tabular}{|c|c|c|c|c|}
\hline \multirow[b]{2}{*}{ Histologic diagnosis } & \multicolumn{2}{|l|}{ FTIR classification, $n$} & \multirow[b]{2}{*}{ Samples/patients, $n$} & \multirow[b]{2}{*}{ Statistical measures, \% } \\
\hline & Positive & Negative & & \\
\hline Cancerous & Correct positive: & False negative: & Cancerous samples: & Sensitivity: \\
\hline \multirow[t]{3}{*}{ Healthy } & False positive: & Correct negative: & Noncancerous samples: & Specificity: \\
\hline & 2 & 39 & 41 & 95.1 \\
\hline & Positive predictive value: & Negative predictive value: & Total: & Accuracy: \\
\hline
\end{tabular}

Data are expressed as $n$, unless otherwise indicated.

FTIR, Fourier transform infrared. 


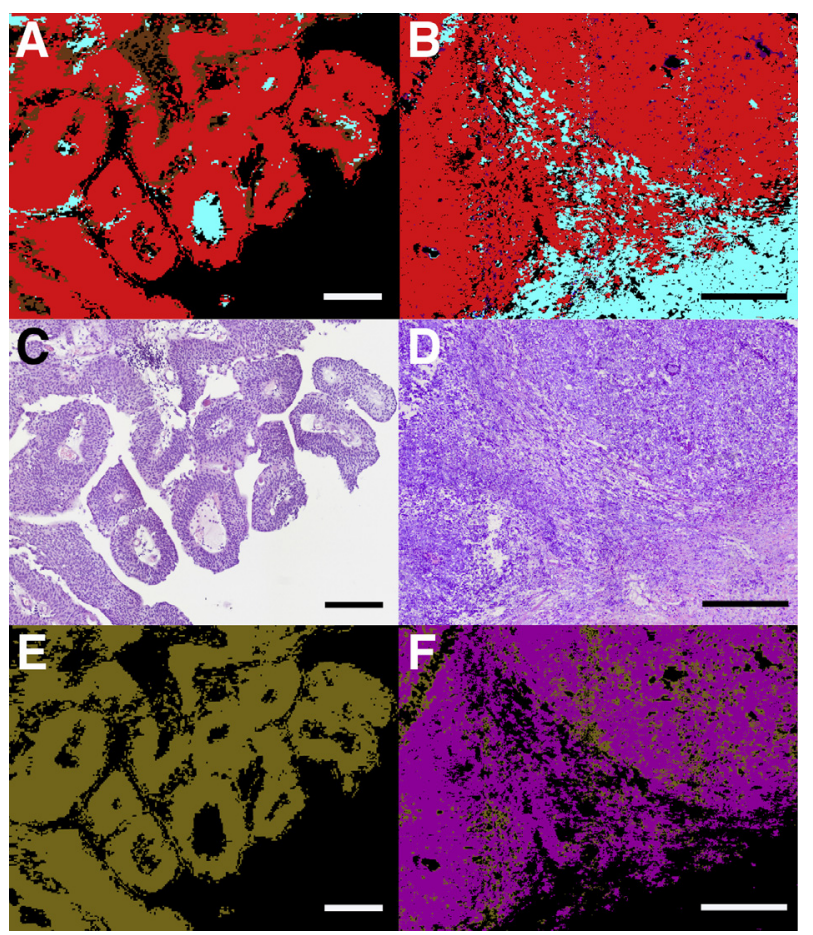

Figure 1 Fourier transform infrared (FTIR) imaging index color images obtained label free before hematoxylin and eosin (H\&E) staining of bladder cancer tissue. A and B: Low-grade and invasive high-grade carcinoma (red, bladder cancer; cyan, connective tissue; brown, inflammation; purple, urothelial cells). C and D: Corresponding H\&E staining after FTIR imaging. The label-free classification matches the cancerous regions. $\mathbf{E}$ and $\mathbf{F}$ : Results of the second random forest, which classified the cancer as invasive highgrade (purple) or low-grade (olive) carcinoma. E: No invasive high-grade carcinoma is observed. Scale bars $=200 \mu \mathrm{m}$.

criterion-which maximized the sum of sensitivity and specificity-was applied to determine the optimal threshold point from an ROC curve.

\section{Results}

\section{FTIR-Guided LCM}

FTIR-guided LCM was used for label-free tissue annotation and harvesting. The spatially resolved FTIR spectra of labelfree, freshly frozen thin tissue sections were used as a molecular fingerprint of the proteome, genome, transcriptome, lipidome, and metabolome of the underlying tissue. ${ }^{26}$ Index colors were assigned to the spectra to classify the tissue by digital labeling on the basis of an RF algorithm. Previously developed bioinformatics workflow was used. ${ }^{32,35}$ This classifier was trained using 11 samples (sample set 1) and verified using 103 freshly frozen samples (sample set 2) that included 41 cystitis, 19 low-grade carcinoma, and 43 invasive high-grade carcinoma. The samples were compared to H\&E-stained images - the clinical gold standard-annotated by a trained pathologist, and showed a specificity of $95 \%$, a sensitivity of $95 \%$, and an accuracy of 95\% (Table 1).
The identified cancer ROIs of the first RF were classified as low grade $(n=18)$ and invasive high grade $(n=41)$ by a second RF with a specificity of $94 \%$, a sensitivity of $93 \%$, and an accuracy of $93 \%$ (Supplemental Table S4) compared with the annotation by the pathologist. The label-free classification was then used to microdissect 10 invasive highgrade $\mathrm{BC}$ and 7 cystitis homogeneous tissue samples (sample set 3). The thin tissue sections were classified label free by FTIR imaging. Homogeneous ROIs in both specimens (cystitis and invasive high grade) were selected, transferred to the LCM, and then microdissected for subsequent proteome analysis, according to a previously described workflow. ${ }^{26}$ The label-free tissue classification is exemplarily shown in Figure 1. The first RF classification differentiated between healthy and cancerous tissue. The cancer tissue is shown in red, connective tissue in cyan, inflammation in brown, and urothelial cells in purple
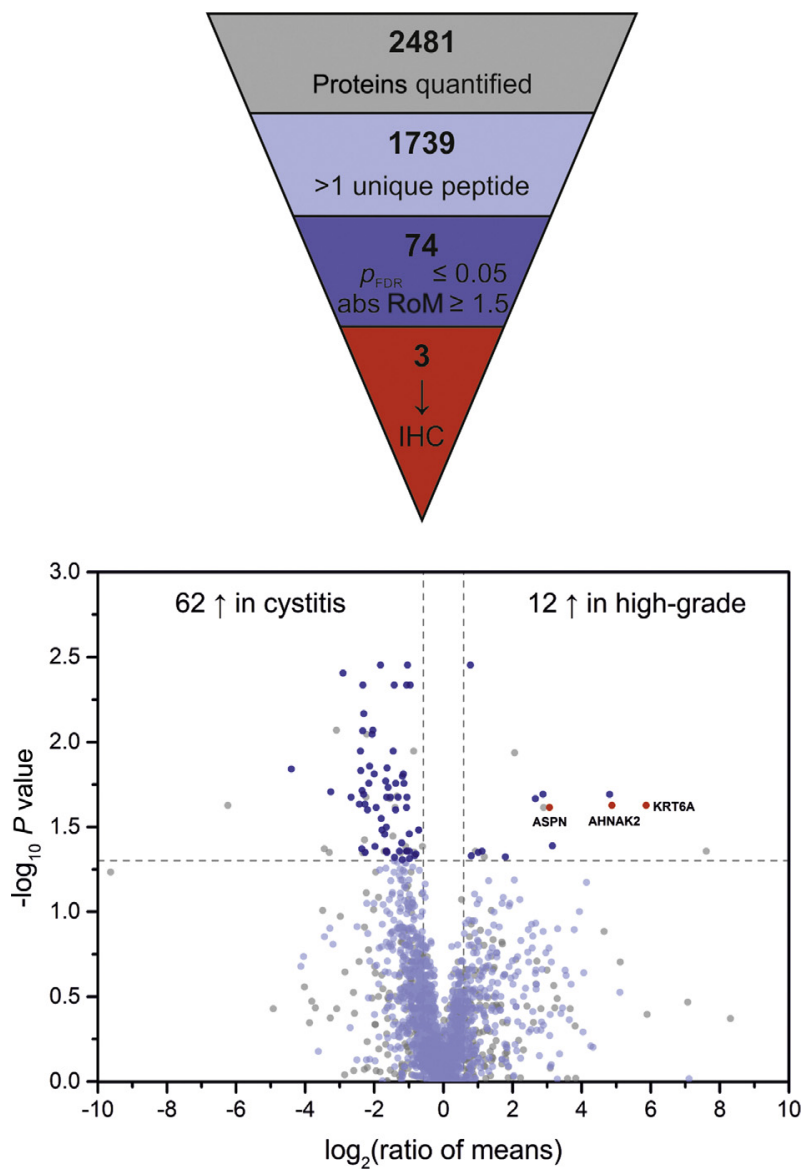

Figure 2 Differential analysis of mass spectrometry data. Thresholds for differentially expressed proteins for $p_{\mathrm{FDR}}$ were 0.05 and 1.5 for absolute ratios of means (RoM), as indicated by gray dashed lines. Positive and negative ratios of means indicate higher protein abundance in invasive high grade and severe cystitis with reactive urothelial atypia, respectively. All quantified proteins are shown. Proteins selected for further verification are annotated and shown in red; differentially expressed proteins are shown in dark purple; proteins with more than one unique peptide are depicted in light purple; and residual quantified proteins with only one unique peptide are depicted in gray. IHC, immunohistochemistry. 
Table 2 Proteins More Highly Expressed in Invasive High-Grade Bladder Cancer Samples

\begin{tabular}{|c|c|c|c|c|}
\hline UniProt ID* & Gene name & Protein name & $p_{\mathrm{FDR}}$ & Ratio of means \\
\hline P02538 & KRT6A & Keratin, type II cytoskeletal 6A & 0.0236 & 58.3 \\
\hline Q8IVF2 & AHNAK2 & Protein AHNAK2 & 0.0236 & 29.4 \\
\hline P04264 & KRT1 & Keratin, type II cytoskeletal 1 & 0.0408 & 8.9 \\
\hline Q9BXN1 & $A S P N$ & Asporin & 0.0243 & 8.4 \\
\hline Q9NR30 & $D D \times 21$ & Nucleolar RNA helicase 2 & 0.0203 & 7.4 \\
\hline Q96RP9 & GFM1 & Elongation factor $\mathrm{G}$, mitochondrial & 0.0438 & 2.2 \\
\hline P41250 & GARS & Glycine-tRNA ligase & 0.0446 & 2.0 \\
\hline 095782 & $A P 2 A 1$ & AP-2 complex subunit $\alpha-1$ & 0.0468 & 1.7 \\
\hline Q00341 & $H D L B P$ & Vigilin & 0.0035 & 1.7 \\
\hline
\end{tabular}

*Searchable at https://www.uniprot.org.

$p_{\mathrm{FDR}}$, FDR-corrected $P$ value.

(Figure 1, A and B). These match the annotation in the corresponding H\&E-stained section (Figure 1, C and D). A second RF to classify the grading further analyzed the cancerous class: low- and invasive high-grade carcinoma (Figure 1, E and F). No invasive high-grade carcinoma was observed in the low-grade carcinoma section, whereas a mixture of low- and invasive high-grade carcinoma was detected in high-grade carcinoma. This is in agreement with histopathology and proves the usability of label-free classification by FTIR imaging.

\section{Quantitative Proteomics Analysis of LCM Tissue Samples}

The next step was an unbiased quantitative proteomic analysis using samples harvested by FTIR-guided LCM. A total of 3515 proteins were identified using Proteome Discoverer software, and 2481 were quantified by Progenesis QI, of which 1739 had more than one unique peptide. There were 74 proteins that showed differential abundance (adjusted $P \leq 0.05$ and absolute ratio of means $\geq 1.5$ ) (Figure 2); of these, 62 were higher abundant in cystitis samples and 12 were more abundant in the invasive high-grade group (Figure 2 and Supplemental Table S5).

\section{Selection of Candidate Biomarkers for Verification}

The 12 proteins that were significantly higher abundant in invasive high-grade tumor samples were analyzed in greater detail as potential biomarkers (Table 2). Several factors were considered for this selection, including the ratio of means or their known/unknown association in the context of bladder cancer.

The proteins with the highest ratios of means [ie, keratin, type II cytoskeletal (KRT)6A and AHNAK2] that were selected for verification were 58.3- and 29.4-fold higher abundant in high-grade cancer compared with cystitis. In contrast, ASPN had no known association with bladder cancer and was therefore included in the verification. Regulation profiles of the candidates are shown in Figure 3.

\section{Evaluation of Three Candidate Biomarkers by IHC}

AHNAK2, KRT6A, and ASPN expression in severe cystitis and low-grade and invasive high-grade bladder cancer specimens were examined by IHC (sample set 4) to assess their potential as biomarkers (Figure 4A). KRT6A was highly expressed in most cystitis and some low-grade cancer cells but was undetectable in invasive high-grade cancer cells. ASPN showed moderate immunoreactivity in many cystitis cells, moderate-to-high expression in all low-grade cancer cells, and high expression in all invasive high-grade cancer cells. AHNAK2 expression was absent in cystitis, with only some background staining in connective tissue, faintly

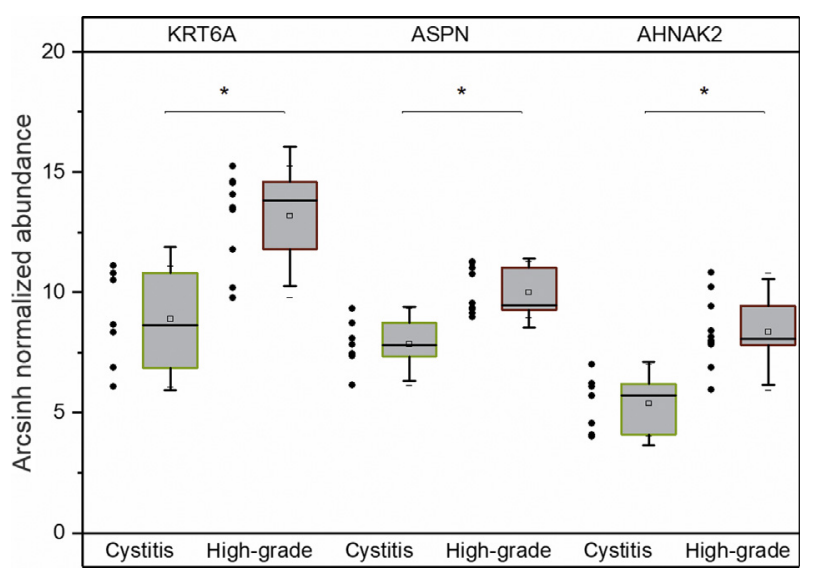

Figure 3 Regulation profiles of selected candidate proteins. Data were transformed to obtain a normal distribution. Boxes represent the 25th and 75th percentiles; whiskers extend to the most extreme data points; the median is shown as a horizontal line; and the mean value is shown as a square within the box. Green boxes indicate cystitis; red boxes, highgrade bladder cancer. ${ }^{*} P \leq 0.05$. 
A

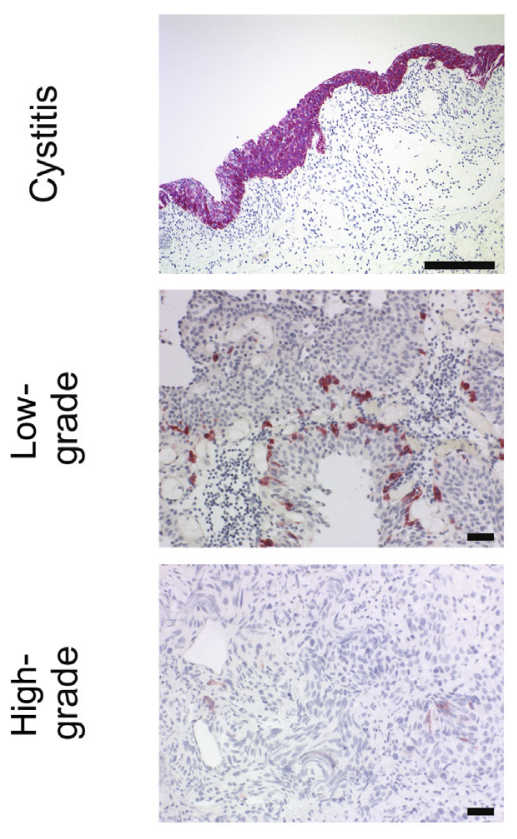

ASPN
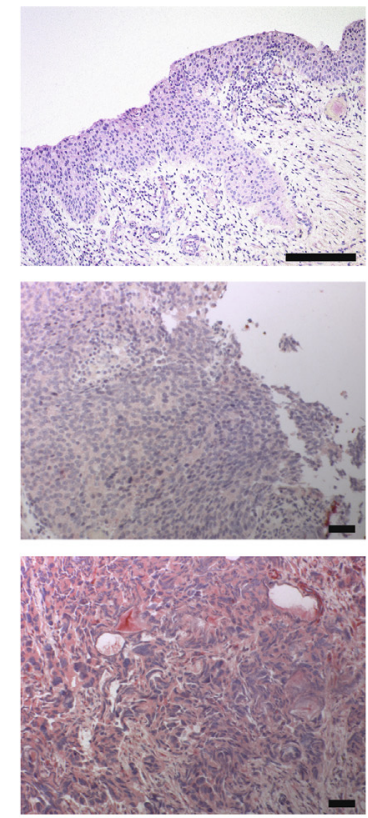

AHNAK2
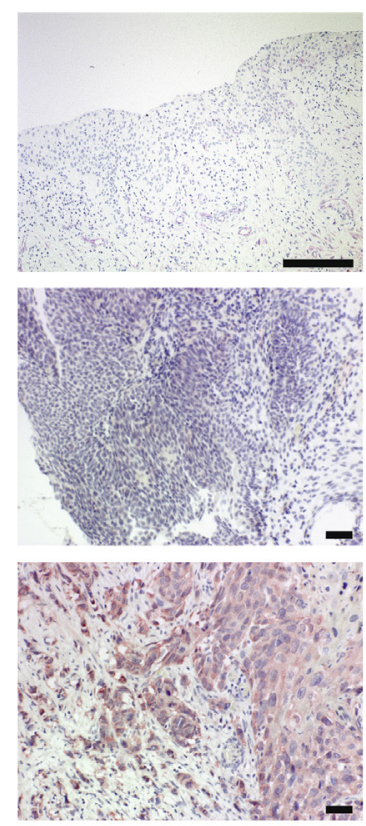
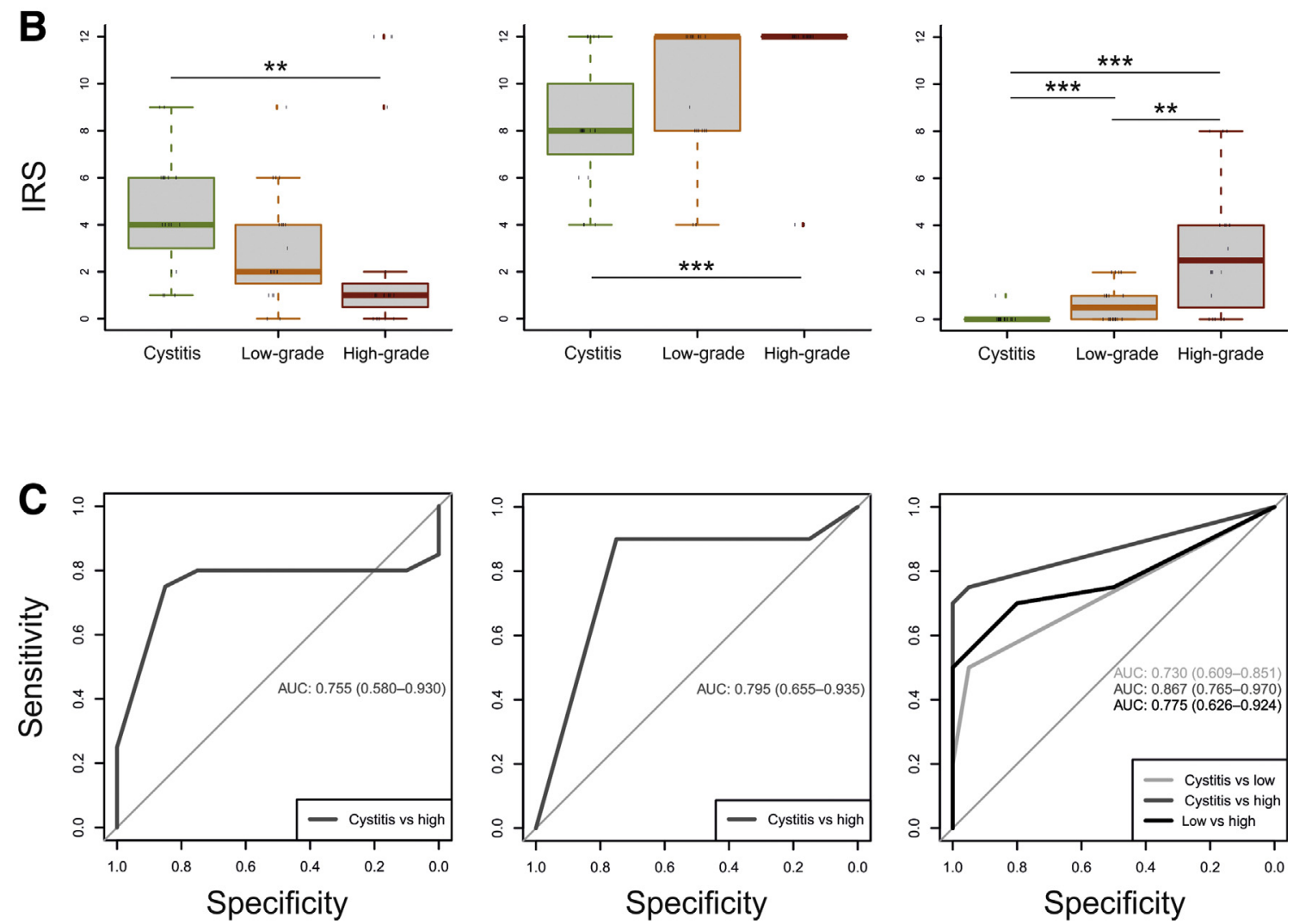

Figure 4 Immunohistochemical verification of AHNAK2, KRT6A, and ASPN. A: Representative staining for three candidate biomarkers in each group (severe cystitis with reactive urothelial atypia (RUA) and low- and invasive high-grade bladder cancer). B: Boxplots of immune reactive score (IRS) for severe cystitis with RUA and low-grade and invasive high-grade bladder cancer. Boxes represent the 25th and 75th percentiles; whiskers extend to the most extreme data points; and the median is shown as a horizontal line. Green indicates cystitis; orange, low-grade bladder cancer; red, high-grade bladder cancer. C: Receiver operating characteristic curves showing best specificity and sensitivity for each marker in comparisons with significant differences. Area under the curve (AUC) is shown, and ranges in parentheses show the $95 \%$ CI. $n=20$ (B, severe cystitis with RUA and low-grade and invasive high-grade bladder cancer). ${ }^{* *} P \leq 0.01,{ }^{* * * P} \leq 0.001$. Scale bars: $200 \mu \mathrm{m}$ (A, top row); $50 \mu \mathrm{m}$ (A, middle and bottom rows). 
A

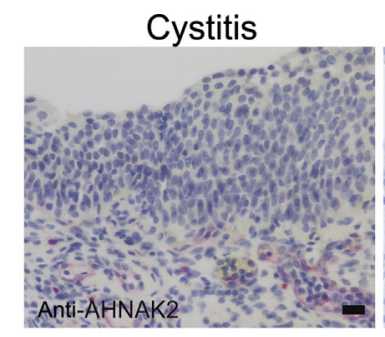

Carcinoma in situ
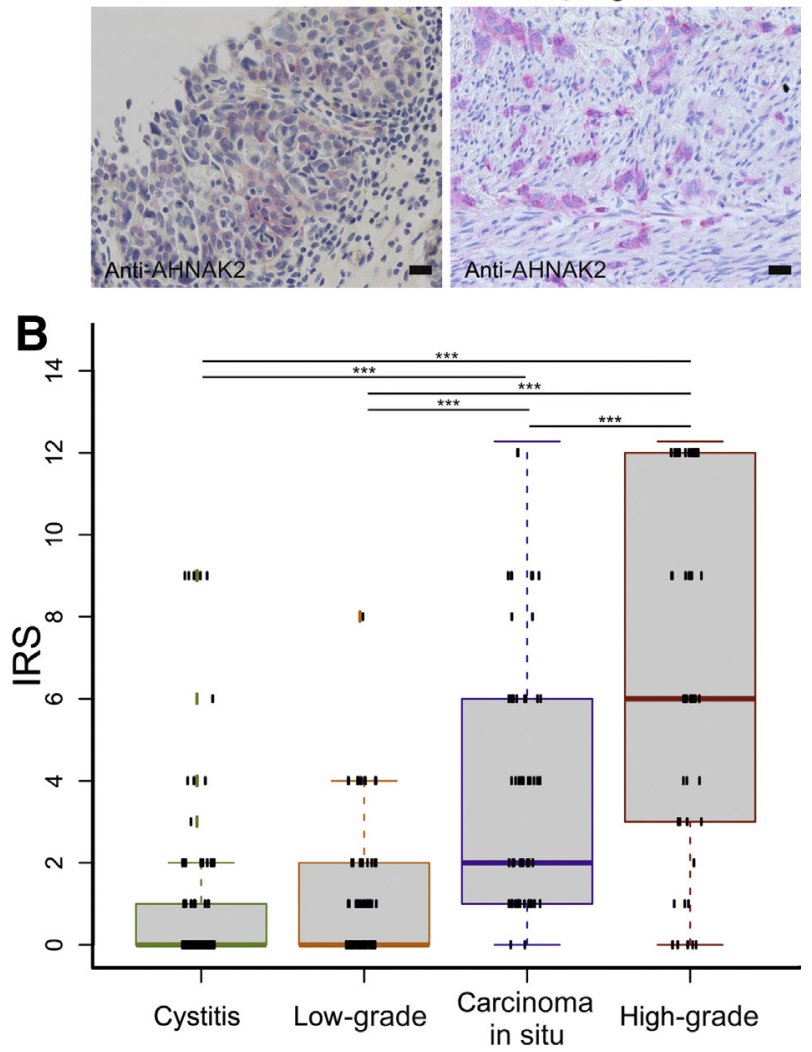

Figure 5 Evaluation of immune reactive score (IRS) for AHNAK2 in the second verification cohort. A: Representative staining for AHNAK2 in each group [severe cystitis with reactive urothelial atypia (RUA), lowgrade and invasive high-grade bladder cancer, and carcinoma in situ]. B: Boxplots display IRS for severe cystitis with RUA, low-grade and invasive high-grade bladder cancer, and carcinoma in situ. Boxes represent the 25th and 75th percentiles; whiskers extend to the most extreme data points; and the median is shown as a horizontal line. Green indicates cystitis; orange, low-grade bladder cancer; blue, carcinoma in situ; red, high-grade bladder cancer. $n=108$ (B, severe cystitis with $\mathrm{RUA}$ ); $n=84$ (B, low-grade bladder cancer); $n=51$ (B, invasive highgrade bladder cancer); $n=67$ (B, carcinoma in situ). ${ }^{* * *} P \leq 0.001$. Scale bars $=20 \mu \mathrm{m}(\mathbf{A})$.

detected in some low-grade bladder cancer cells, and high in many invasive high-grade bladder cancer cells.

An IRS - which encompasses both the percentage of stained cells and staining intensity - was assigned to each sample for each of the candidate biomarkers (Figure 4B). Only AHNAK2 expression showed significant differences between all three groups (Supplemental Table S6). ROC curves were generated to evaluate the relationship between sensitivity (true positive rate) and specificity (true negative rate) of the markers (Supplemental Table S7). The largest area under the curve for the comparison between cystitis and invasive high-grade bladder cancer was obtained for AHNAK2 (0.87), with a sensitivity of $75 \%$ and a specificity of $95 \%$ (Figure 4C). By comparison, ASPN has a smaller area under the curve $(0.80)$, with a sensitivity and a specificity of $90 \%$ and $75 \%$, respectively; and KRT6A had the smallest area under the curve (0.76), with a sensitivity and a specificity of $75 \%$ and $85 \%$, respectively. All other comparisons were only significant for AHNAK2, and this protein was therefore selected for an additional verification step in a larger cohort that included a CIS group.

\section{Second Verification Step for AHNAK2}

AHNAK2 was further verified using FFPE tissue samples from a larger, independent patient cohort comprising four groups, including severe cystitis with RUA $(n=108)$, invasive high grade with no concomitant CIS $(n=51)$, CIS $(n=67)$, and low grade $(n=84)$. AHNAK2 expression (Figure 5A) was, again, absent in cystitis, with only some background staining in connective tissue, not detectable in most low-grade bladder cancer samples, well detectable in carcinoma in situ, and high in many high-grade bladder cancer cells. AHNAK2 levels differed most significantly between RUA and CIS, and between RUA and invasive high grade (Figure 5B and Supplemental Table S8). The ROC curves between RUA and CIS (Figure 6A), low and invasive high grades (Figure 6B), and low grade and CIS (Figure 6C) revealed that for the differentiation between RUA and CIS, AHNAK2 had a sensitivity of $97 \%$ and a specificity of $69 \%$ at a cutoff value of 0.5 ; for differentiating between low and high grades, the sensitivity and specificity were $80 \%$ and $86 \%$, respectively, at a cutoff value of 2.5 ; and for differentiating between low-grade carcinoma and CIS, the sensitivity and specificity were $97 \%$ and $55 \%$, respectively, at a cutoff value of 0.5 . All other comparisons are shown in Supplemental Table S9 and Supplemental Figure S1.

\section{Discussion}

There is a need for a sensitive and specific biomarker for bladder cancer because of high interobserver variability in established diagnostic approaches, ${ }^{47-52}$ which can lead to a failure to distinguish between histologically similar subtypes. In particular, differentiating between reactive urothelial atypia in the setting of acute or chronic urocystitis or low-grade nonmuscle invasive-type bladder cancer and the noninvasive but high-grade CIS is important for therapeutic decision making. Because these entities may be histologically similar to each other but show immensely different prognoses, they can mean a diagnostic dilemma. Tumor grade is an important prognostic factor for risk stratification, and distinguishing between low- and invasive high-grade bladder cancer is 

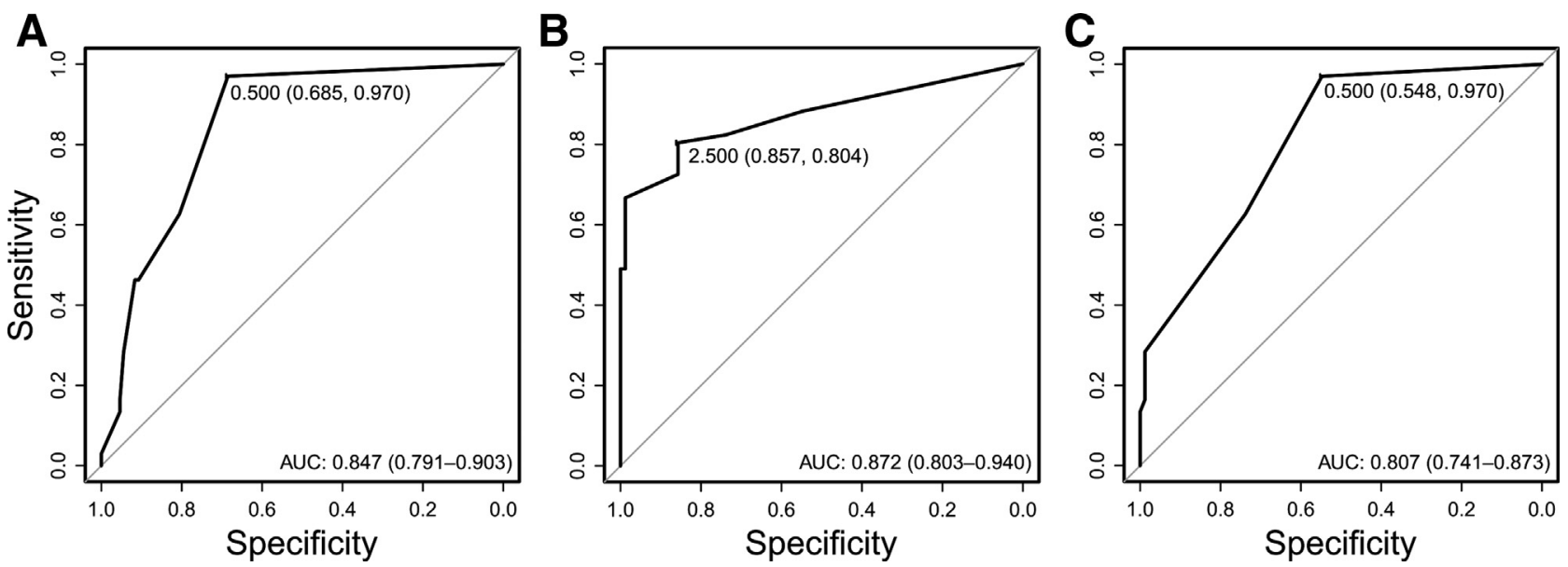

Figure 6 Statistical analysis of immune reactive score (IRS) determined from receiver operating characteristic (ROC) curves. ROC curves show Specificity and sensitivity for each evaluated comparison. A: Severe cystitis with reactive urothelial atypia versus carcinoma in situ (CIS). B: Differentiation between low grade and invasive high grades. C: Comparison between low grade and CIS. IRS cutoff values are indicated at the turning point of the curve, with specificity and sensitivity values shown in parentheses. Area under the curve (AUC) is shown, and ranges in parentheses indicate the $95 \%$ CI.

clinically important. At present, there are no reliable IHC biomarkers in regular clinical use that can aid pathologists in difficult cases, whose only option in cases of uncertainty is to seek a second opinion from their peers.

To identify novel candidate biomarkers, an unbiased approach integrating label-free automated tissue annotation and global label-free LC-MS/MS proteomic analysis was used. The material of choice for the discovery phase was freshly frozen tissue; as such, the analyzed disease stages in this phase were limited to severe urocystitis and invasive high-grade bladder cancer without accompanying CIS. Tissue biopsies obtained by cystectomy in the case of invasive high-grade bladder cancer are suitable for LCM with subsequent proteome analysis, unlike low-grade bladder cancer that is usually only obtained in small amounts by transurethral resection. ${ }^{53}$ However, cystectomy samples tend to contain large regions of invasive high-grade carcinoma and cystitis with RUA, which were analyzed in the discovery phase of the present study.

Label-free FTIR imaging identifies not only the cancer region as would an IHC marker panel, but also all other tissue types, including a clear depiction of regions of inflammation. Multiplex FTIR imaging thus replaces not only a single biomarker but also a multi-IHC panel. ${ }^{26}$ FTIRguided LCM allows label-free, observer-independent, and precise harvesting of homogeneous tissue for subsequent LC-MS/MS analysis.

The proteomic analysis identified three potential biomarkers for bladder cancer. Despite the fact of promising biomarker candidates, also for bladder cancer, in which lower abundance correlates to tumor progression, ${ }^{54}$ only proteins higher abundant in bladder cancer were considered for further analysis. This is attributed to the fact that the discovery cohort consisted of severe urocystitis and invasive high-grade bladder cancer samples, in which LCM provided homogeneous samples not confounded by tissue other than stated. However, high-grade bladder cancer is often accompanied by areas of urocystitis, which could confound the biomarker results in clinical routine if the marker is more highly abundant in urocystitis samples. In addition, although negative biomarkers also provide valuable biological information, evaluation of positive biomarkers, which stain the diseased state, is much easier in clinical routine. Although KRT6A and AHNAK2 were selected on the basis of their high ratios of means, ASPN was chosen because it has not yet been linked to bladder cancer. The candidate proteins were verified by the wellestablished method of IHC using FFPE tissue. The advantage herein was the inclusion of additional bladder cancer grades because FFPE tissue is suitable for IHC analysis irrespective of the method of tissue collection. Therefore, low-grade samples and cystitis samples containing reactive urothelial atypia were included in the first verification step in a small cohort of 20 samples per group. Of the three candidate proteins, only AHNAK2 showed significant differences in all comparisons and, more important, in the comparison of low- versus invasive high-grade bladder cancer. Therefore, only AHNAK2 was retained for the second verification step with a larger patient cohort that included CIS samples. Discrepancies between the results of the verification step and proteomics data, as observed for KRT6A, can be attributed to random biological variance given that two different patient cohorts were used for identification and subsequent verification. An alternative explanation is that in bladder cancer, keratin $6 \mathrm{~A}$ is overexpressed only in malignant cells with activation of growth factor signaling. ${ }^{55}$ This could be true of invasive high-grade cases in the discovery study and for cases with high IRSs in the verification of KRT6A by IHC.

In this study, AHNAK2 was identified and verified in two steps as a candidate biomarker for bladder cancer. AHNAK2 is a $600-\mathrm{kDa}$ protein with a high number of 
conserved repeats; although its function is largely unknown, it is thought to associate with calcium channel proteins. ${ }^{56}$ AHNAK2 has already been proposed as a potential prognostic biomarker for clear renal cell and pancreatic cancers, ${ }^{57,58}$ and is part of a urinary mRNA panel for the diagnosis of bladder cancer and prediction of tumor aggressiveness. ${ }^{59,60}$ In the present study, AHNAK2 showed promise for differentiating between low- and invasive high-grade muscle invasive and noninvasive cancer (CIS), and especially between the oftentimes hard-todistinguish RUA and CIS. ${ }^{8}$ A sensitivity of $97 \%$ was achieved for the discrimination between cystitis and CIS and between low grade and CIS, with specificities of $69 \%$ and 55\%, respectively. Misdiagnosis of CIS can delay treatment of an aggressive malignancy ${ }^{61}$ or could lead to unnecessary treatment by intravesical instillation therapy with Bacillus Calmette-Guèrin or even early radical cystectomy (the recommended treatment option for Bacillus Calmette-Guèrin-refractory CIS), ${ }^{4}$ with all of the associated adverse effects. ${ }^{62-64}$ AHNAK2 could be a helpful tool for detecting CIS recurrence or persistence after Bacillus Calmette-Guèrin therapy for CIS, in which an inflammatory response is deliberately induced in the bladder. ${ }^{65,66}$ Although the cutoff value in both cases was low at 0.5 , differentiation was usually possible because most cystitis and low-grade cases had an IRS of 0 . There were a few outliers in the RUA cohort with IRSs of up to 9, but in at least one of these cases, we had follow-up information that the patient was diagnosed with low-grade bladder cancer 6 months later. This suggests that AHNAK2 could serve as a prognostic biomarker, which should be investigated in future studies. AHNAK2 had a sensitivity of $80 \%$ and a specificity of $86 \%$ for the differentiation between low- and invasive high-grade bladder cancer, demonstrating that it can be useful for therapeutic decision making by pathologists in borderline cases.

Besides its detection by IHC in tissue sections, AHNAK2 should be considered as a urinary marker because there are no others that are accepted for diagnosis or follow-up in routine practice or in clinical guidelines. Some urinary biomarker tests have been proposed for bladder cancer detection, such as the protein-based BTA STAT or BTA TRAK assays (both from POLYMEDCO, New York, NY) or NMP22BladderChek/NMP22 (Alere, Scarborough, ME), and the cell-based UroVysion (Abbott Molecular, Inc., Des Plaines, IL) or ImmunoCyt/uCyt+ (Scimedx Corp., Denville, $\mathrm{NJ}$ ) test that allow noninvasive diagnosis of bladder cancers in contrast to invasive cytologic methods. ${ }^{67-72}$ However, all these tests yield results with high variance and are therefore not recommended by clinical guidelines. A study is currently underway to evaluate the diagnostic value of AHNAK2 in cytologic analyses. However, the results demonstrate that AHNAK2 could be a useful candidate biomarker for IHC that can be used as an adjunct to H\&E staining in cases in which it is difficult to differentiate between bladder cancer subtypes.
Overall, the FTIR imaging approach described herein achieves high sensitivity and specificity for tissue classification in bladder cancer and allows precise selection of ROIs for subsequent proteomics-based analyses. This novel approach can be extended to other cancer types to provide new biomarker candidates.

\section{Acknowledgments}

The authors would like to thank Lidia Janota, Kristin Rosowski, Stephanie Tautges, and Birgit Zülch for their excellent technical assistance.

\section{Supplemental Data}

Supplemental material for this article can be found at https://doi.org/10.1016/j.ajpath.2018.11.018.

\section{References}

1. Antoni S, Ferlay J, Soerjomataram I, Znaor A, Jemal A, Bray F: Bladder cancer incidence and mortality: a global overview and recent trends. Eur Urol 2017, 71:96-108

2. Ferlay J, Soerjomataram I, Dikshit R, Eser S, Mathers C, Rebelo M, Parkin DM, Forman D, Bray F: Cancer incidence and mortality worldwide: sources, methods and major patterns in GLOBOCAN 2012. Int J Cancer 2015, 136:E359-E386

3. Ye F, Wang L, Castillo-Martin M, McBride R, Galsky MD, Zhu J, Boffetta P, Zhang DY, Cordon-Cardo C: Biomarkers for bladder cancer management: present and future. Am J Clin Exp Urol 2014, 2:1-14

4. Babjuk M, Böhle A, Burger M, Capoun O, Cohen D, Compérat EM, Hernández V, Kaasinen E, Palou J, Rouprêt M, van Rhijn BWG, Shariat SF, Soukup V, Sylvester RJ, Zigeuner R: EAU guidelines on non-muscle-invasive urothelial carcinoma of the bladder: update 2016. Eur Urol 2017, 71:447-461

5. Kirkali Z, Chan T, Manoharan M, Algaba F, Busch C, Cheng L, Kiemeney L, Kriegmair M, Montironi R, Murphy WM, Sesterhenn IA, Tachibana M, Weider J: Bladder cancer: epidemiology, staging and grading, and diagnosis. Urology 2005, 66:4-34

6. Knowles MA, Hurst CD: Molecular biology of bladder cancer: new insights into pathogenesis and clinical diversity. Nat Rev Cancer 2015, 15:25-41

7. Pow-Sang JM, Seigne JD: Contemporary management of superficial bladder cancer. Cancer Control 2000, 7:335-339

8. Lamm D, Herr H, Jakse G, Kuroda M, Mostofi FK, Okajima E, Sakamoto A, Sesterhenn I, da Silva FC: Updated concepts and treatment of carcinoma in situ. Urol Oncol 1998, 4:130-138

9. Sternberg CN: Muscle invasive and metastatic bladder cancer. Ann Oncol 2006, 17 Suppl 10:x23-x30

10. Witjes JA, Compérat E, Cowan NC, De Santis M, Gakis G, Lebret T, Ribal MJ, Van der Heijden AG, Sherif A; European Association of Urology: EAU guidelines on muscle-invasive and metastatic bladder cancer: summary of the 2013 guidelines. Eur Urol 2014, 65:778-792

11. Rizzardi AE, Johnson AT, Vogel RI, Pambuccian SE, Henriksen J, Skubitz AP, Metzger GJ, Schmechel SC: Quantitative comparison of immunohistochemical staining measured by digital image analysis versus pathologist visual scoring. Diagn Pathol 2012, 7:42

12. Witjes JA, Moonen PMJ, van der Heijden AG: Review pathology in a diagnostic bladder cancer trial: effect of patient risk category. Urology 2006, 67:751-755

13. Chen C-L, Lin T-S, Tsai C-H, Wu C-C, Chung T, Chien K-Y, Wu M, Chang Y-S, Yu J-S, Chen Y-T: Identification of potential bladder 
cancer markers in urine by abundant-protein depletion coupled with quantitative proteomics. J Proteomics 2013, 85:28-43

14. Lei T, Zhao X, Jin S, Meng Q, Zhou H, Zhang M: Discovery of potential bladder cancer biomarkers by comparative urine proteomics and analysis. Clin Genitourin Cancer 2013, 11:56-62

15. Bansal N, Gupta A, Sankhwar SN, Mahdi AA: Low- and high-grade bladder cancer appraisal via serum-based proteomics approach. Clin Chim Acta 2014, 436:97-103

16. Schwamborn K, Krieg RC, Grosse J, Reulen N, Weiskirchen R, Knuechel R, Jakse G, Henkel C: Serum proteomic profiling in patients with bladder cancer. Eur Urol 2009, 56:989-996

17. Feldman AS, Banyard J, Wu C-L, McDougal WS, Zetter BR: Cystatin B as a tissue and urinary biomarker of bladder cancer recurrence and disease progression. Clin Cancer Res 2009, 15: 1024-1031

18. Schmitz-Dräger BJ, Goebell PJ, Ebert T, Fradet Y: p53 Immunohistochemistry as a prognostic marker in bladder cancer: playground for urology scientists? Eur Urol 2000, 38:691-699; discussion 700

19. Harnden P, Mahmood N, Southgate J: Expression of cytokeratin 20 redefines urothelial papillomas of the bladder. Lancet 1999, 353: 974-977

20. Asgari M, Nabi Maybodi M, Abolhasani M: Differential diagnosis of urothelial carcinoma in situ from non-neoplastic urothelia: analysis of CK20, CD44, P53 and Ki67. Med J Islam Repub Iran 2016, 30:400

21. Padden J, Ahrens M, Kalsch J, Bertram S, Megger DA, Bracht T, Eisenacher M, Kocabayoglu P, Meyer HE, Sipos B, Baba HA, Sitek B: Immunohistochemical markers distinguishing cholangiocellular carcinoma (CCC) from pancreatic ductal adenocarcinoma (PDAC) discovered by proteomic analysis of microdissected cells. Mol Cell Proteomics 2016, 15:1072-1082

22. Xiao H, Langerman A, Zhang Y, Khalid O, Hu S, Cao C-X, Lingen MW, Wong DTW: Quantitative proteomic analysis of microdissected oral epithelium for cancer biomarker discovery. Oral Oncol 2015, 51:1011-1019

23. Liu NQ, Dekker LJM, Stingl C, Güzel C, De Marchi T, Martens JWM, Foekens JA, Luider TM, Umar A: Quantitative proteomic analysis of microdissected breast cancer tissues: comparison of label-free and SILAC-based quantification with shotgun, directed, and targeted MS approaches. J Proteome Res 2013, 12:4627-4641

24. Li M, Peng F, Li G, Fu Y, Huang Y, Chen Z, Chen Y: Proteomic analysis of stromal proteins in different stages of colorectal cancer establishes tenascin-C as a stromal biomarker for colorectal cancer metastasis. Oncotarget 2016, 7:37226-37237

25. Chen C-L, Chung T, Wu C-C, Ng K-F, Yu J-S, Tsai C-H, Chang Y-S, Liang Y, Tsui K-H, Chen Y-T: Comparative tissue proteomics of microdissected specimens reveals novel candidate biomarkers of bladder cancer. Mol Cell Proteomics 2015, 14:2466-2478

26. Großerueschkamp F, Bracht T, Diehl HC, Kuepper C, Ahrens M, Kallenbach-Thieltges A, Mosig A, Eisenacher M, Marcus K, Behrens T, Brüning T, Theegarten D, Sitek B, Gerwert K: Spatial and molecular resolution of diffuse malignant mesothelioma heterogeneity by integrating label-free FTIR imaging, laser capture microdissection and proteomics. Sci Rep 2017, 7:44829

27. Bhargava R: Infrared spectroscopic imaging: the next generation. Appl Spectrosc 2012, 66:1091-1120

28. Hughes C, Iqbal-Wahid J, Brown M, Shanks JH, Eustace A, Denley H, Hoskin PJ, West C, Clarke NW, Gardner P: FTIR microspectroscopy of selected rare diverse sub-variants of carcinoma of the urinary bladder. J Biophoton 2013, 6:73-87

29. Diem M, Mazur A, Lenau K, Schubert J, Bird B, Miljković M, Krafft C, Popp J: Molecular pathology via IR and Raman spectral imaging. J Biophoton 2013, 6:855-886

30. Pilling M, Gardner P: Fundamental developments in infrared spectroscopic imaging for biomedical applications. Chem Soc Rev 2016, 45:1935-1957
31. Krafft C, Steiner G, Beleites C, Salzer R: Disease recognition by infrared and Raman spectroscopy. J Biophoton 2009, 2:13-28

32. Großerueschkamp F, Kallenbach-Thieltges A, Behrens T, Brüning T, Altmayer M, Stamatis G, Theegarten D, Gerwert K: Marker-free automated histopathological annotation of lung tumour subtypes by FTIR imaging. Analyst 2015, 140:2114-2120

33. Miller LM, Dumas P: Chemical imaging of biological tissue with synchrotron infrared light. Biochim Biophys Acta 2006, 1758: 846-857

34. Ooi GJ, Fox J, Siu K, Lewis R, Bambery KR, McNaughton D, Wood BR: Fourier transform infrared imaging and small angle $\mathrm{x}$-ray scattering as a combined biomolecular approach to diagnosis of breast cancer. Med Phys 2008, 35:2151-2161

35. Kallenbach-Thieltges A, Großerüschkamp F, Mosig A, Diem M, Tannapfel A, Gerwert K: Immunohistochemistry, histopathology and infrared spectral histopathology of colon cancer tissue sections. J Biophoton 2013, 6:88-100

36. Bassan P, Byrne HJ, Bonnier F, Lee J, Dumas P, Gardner P: Resonant Mie scattering in infrared spectroscopy of biological materials: understanding the "dispersion artefact". Analyst 2009, 134:1586-1593

37. Bassan P, Kohler A, Martens H, Lee J, Byrne HJ, Dumas P, Gazi E, Brown M, Clarke N, Gardner P: Resonant Mie Scattering (RMieS) correction of infrared spectra from highly scattering biological samples. Analyst 2010, 135:268-277

38. Savitzky A, Golay MJE: Smoothing and differentiation of data by simplified least squares procedures. Anal Chem 1964, 36:1627

39. Bracht T, Schweinsberg V, Trippler M, Kohl M, Ahrens M, Padden J, Naboulsi W, Barkovits K, Megger DA, Eisenacher M, Borchers CH, Schlaak JF, Hoffmann A-C, Weber F, Baba HA, Meyer HE, Sitek B: Analysis of disease-associated protein expression using quantitative proteomics-fibulin-5 is expressed in association with hepatic fibrosis. J Proteome Res 2015, 14:2278-2286

40. Megger DA, Bracht T, Kohl M, Ahrens M, Naboulsi W, Weber F, Hoffmann A-C, Stephan C, Kuhlmann K, Eisenacher M, Schlaak JF, Baba HA, Meyer HE, Sitek B: Proteomic differences between hepatocellular carcinoma and nontumorous liver tissue investigated by a combined gel-based and label-free quantitative proteomics study. Mol Cell Proteomics 2013, 12:2006-2020

41. Papathanassiou D, Etard O, Mellet E, Zago L, Mazoyer B, TzourioMazoyer N: A common language network for comprehension and production: a contribution to the definition of language epicenters with PET. Neuroimage 2000, 11:347-357

42. Benjamini Y, Hochberg Y: Controlling the false discovery rate: a practical and powerful approach to multiple testing. J R Stat Soc Series B Stat Methodol 1995, 57:289-300

43. Mayer G, Stephan C, Meyer HE, Kohl M, Marcus K, Eisenacher M: ProCon: PROteomics CONversion tool. J Proteomics 2015, 129: $56-62$

44. Tannapfel A, Grund D, Katalinic A, Uhlmann D, Kckerling F, Haugwitz U, Wasner M, Hauss J, Engeland K, Wittekind C: Decreased expression of $\mathrm{p} 27$ protein is associated with advanced tumor stage in hepatocellular carcinoma. Int J Cancer 2000, 89: 350-355

45. Remmele W, Stegner HE: Recommendation for uniform definition of an immunoreactive score (IRS) for immunohistochemical estrogen receptor detection (ER-ICA) in breast cancer tissue. Pathologe 1987, 8:138-140

46. Robin X, Turck N, Hainard A, Tiberti N, Lisacek F, Sanchez J-C, Müller M: pROC: an open-source package for $\mathrm{R}$ and $\mathrm{S}+$ to analyze and compare ROC curves. BMC Bioinformatics 2011, 12:77

47. Humphrey PA, Moch H, Cubilla AL, Ulbright TM, Reuter VE: The 2016 WHO classification of tumours of the urinary system and male genital organs, part B: prostate and bladder tumours. Eur Urol 2016 , 70:106-119

48. Bol MG, Baak JP, Buhr-Wildhagen S, Kruse AJ, Kjellevold KH, Janssen EA, Mestad O, Øgreid P: Reproducibility and prognostic 
variability of grade and lamina propria invasion in stages Ta, T1 urothelial carcinoma of the bladder. J Urol 2003, 169:1291-1294

49. Coblentz TR, Mills SE, Theodorescu D: Impact of second opinion pathology in the definitive management of patients with bladder carcinoma. Cancer 2001, 91:1284-1290

50. Lee MC, Levin HS, Jones JS: The role of pathology review of transurethral bladder tumor resection specimens in the modern era. $\mathrm{J}$ Urol 2010, 183:921-928

51. Tosoni I, Wagner U, Sauter G, Egloff M, Knönagel H, Alund G, Bannwart F, Mihatsch MJ, Gasser TC, Maurer R: Clinical significance of interobserver differences in the staging and grading of superficial bladder cancer. BJU Int 2000, 85:48-53

52. Cheng L, Bostwick DG: Overdiagnosis of bladder carcinoma. Anal Quant Cytol Histol 2008, 30:261-264

53. Richards KA, Smith ND, Steinberg GD: The importance of transurethral resection of bladder tumor in the management of nonmuscle invasive bladder cancer: a systematic review of novel technologies. J Urol 2014, 191:1655-1664

54. Boiteux G, Lascombe I, Roche E, Plissonnier M-L, Clairotte A, Bittard H, Fauconnet S, FABP A-: a candidate progression marker of human transitional cell carcinoma of the bladder, is differentially regulated by PPAR in urothelial cancer cells. Int J Cancer 2009, 124: $1820-1828$

55. Somji S, Bathula CS, Zhou XD, Sens MA, Sens DA, Garrett SH: Transformation of human urothelial cells (UROtsa) by as and cd induces the expression of keratin 6a. Environ Health Perspect 2008, 116:434-440

56. Komuro A, Masuda Y, Kobayashi K, Babbitt R, Gunel M, Flavell RA, Marchesi VT: The AHNAKs are a class of giant propeller-like proteins that associate with calcium channel proteins of cardiomyocytes and other cells. Proc Natl Acad Sci U S A 2004, 101: 4053-4058

57. Wang M, Li X, Zhang J, Yang Q, Chen W, Jin W, Huang Y-R, Yang R, Gao W-Q: AHNAK2 is a novel prognostic marker and oncogenic protein for clear cell renal cell carcinoma. Theranostics 2017, 7:1100-1113

58. Lu D, Wang J, Shi X, Yue B, Hao J: AHNAK2 is a potential prognostic biomarker in patients with PDAC. Oncotarget 2017, 8: 31775-31784

59. Noon AP, Zlotta AR: Urothelial bladder cancer urinary biomarkers. EJIFCC 2014, 25:99-114

60. Mengual L, Burset M, Ribal MJ, Ars E, Marín-Aguilera M, Fernández M, Ingelmo-Torres M, Villavicencio H, Alcaraz A: Gene expression signature in urine for diagnosing and assessing aggressiveness of bladder urothelial carcinoma. Clin Cancer Res 2010, 16: $2624-2633$
61. Cheng L, Cheville JC, Neumann RM, Leibovich BC, Egan KS, Spotts BE, Bostwick DG: Survival of patients with carcinoma in situ of the urinary bladder. Cancer 1999, 85:2469-2474

62. Roghmann F, Trinh Q-D, Braun K, von Bodman C, Brock M, Noldus J, Palisaar J: Standardized assessment of complications in a contemporary series of European patients undergoing radical cystectomy. Int J Urol 2014, 21:143-149

63. Shabsigh A, Korets R, Vora KC, Brooks CM, Cronin AM, Savage C, Raj G, Bochner BH, Dalbagni G, Herr HW, Donat SM: Defining early morbidity of radical cystectomy for patients with bladder cancer using a standardized reporting methodology. Eur Urol 2009, 55:164-174

64. Suzuki S, Shinohara N, Harabayashi T, Taniguchi A, Haga K, Sato S, Sakamoto K, Koyanagi T: Complications of bacillus Calmette-Guerin therapy in superficial urothelial cancer: clinical analysis and implications. Int J Clin Oncol 2002, 7:289-293

65. Lamm DL, van der Meijden APM, Morales A, Brosman SA, Catalona WJ, Herr HW, Soloway MS, Steg A, Debruyne FMJ Incidence and treatment of complications of Bacillus Calmette-Guerin intravesical therapy in superficial bladder cancer. J Urol 1992, 147: 596-600

66. Paterson DL, Patel A: Bacillus Calmette-Guerin (BCG) immunotherapy for bladder cancer: review of complications and their treatment. ANZ J Surg 1998, 68:340-344

67. Landman J, Chang Y, Kavaler E, Droller MJ, Liu BC-S: Sensitivity and specificity of NMP-22, telomerase, and BTA in the detection of human bladder cancer. Urology 1998, 52:398-402

68. Thomas L, Leyh H, Marberger M, Bombardieri E, Bassi P, Pagano F, Pansadoro V, Sternberg CN, Boccon-Gibod L, Ravery V, Le Guludec D, Meulemans A, Conort P, Ishak L: Multicenter trial of the quantitative BTA TRAK assay in the detection of bladder cancer. Clin Chem 1999, 45:472-477

69. Kinders R, Jones T, Root R, Bruce C, Murchison H, Corey M, Williams L, Enfield D, Hass GM: Complement factor $\mathrm{H}$ or a related protein is a marker for transitional cell cancer of the bladder. Clin Cancer Res 1998, 4:2511-2520

70. Ellis WJ, Blumenstein BA, Ishak LM, Enfield DL: Clinical evaluation of the BTA trak assay and comparison to voided urine cytology and the bard BTA test in patients with recurrent bladder tumors. Urology 1997, 50:882-887

71. Hajdinjak T: UroVysion FISH test for detecting urothelial cancers: meta-analysis of diagnostic accuracy and comparison with urinary cytology testing. Urol Oncol 2008, 26:646-651

72. Comploj E, Mian C, Ambrosini-Spaltro A, Dechet C, Palermo S, Trenti E, Lodde M, Horninger W, Pycha A: uCyt+/ImmunoCyt and cytology in the detection of urothelial carcinoma. An update on 7422 analyses. Cancer Cytopathol 2013, 121:392-397 